\title{
EFFECT OF HIGH INTENSITY INTERVAL TRAINING ON THE CARDIOVASCULAR SYSTEM AND CARDIAC ANTI-AGING
}

\author{
Olivia Dharmasanti ${ }^{1}$, Shinta Setiono ${ }^{1}$, Johnny B. Nugroho ${ }^{1}$, Suci Nirmala ${ }^{1}$, Goklas R.P. \\ Tambunan $^{1}$, Melindarani Pradita ${ }^{1}$, Yovita Adelia ${ }^{1}$, Indira Vidiari Juhanna ${ }^{2}$ \\ ${ }^{1}$ Anti Aging Medicine Post Graduate Program Biomedic Medical Faculty Universitas Udayana, 80234, \\ Denpansar, Indonesia \\ ${ }^{2}$ Physiology Department Medical Faculty Universitas Udayana, 80234, Denpasar, Indonesia \\ Email : doctressolivie@gmail.com
}

\begin{abstract}
There are a few danger factors prompting the turn of events and movement of CVD, yet one of the most noticeable is an inactive way of life. A solid way of life can be described by both stoutness and reliably low degrees of actual movement. Way of life intercessions that expect to increment actual movement and diminishing stoutness are appealing restorative strategies to battle most non-innate sorts of CVD. Maturing is another significant risk factor for cardio-vascular diseases (CVD). Epidemiological examinations unmistakably show that maturing itself is the significant danger factor for cardiovascular and cerebrovascular illnesses. High-intensity interval training (HIIT) is as often as possible utilized in sports preparing. The impacts on cardiorespiratory and muscle frameworks have driven researchers to think about its application in the field of cardiovascular sicknesses. HIIT is characterized as extreme focus practice for a characterized timeframe sprinkled with rest periods (moderate light intensity or complete rest). This literature review was conducted to determine the correlation between high intensity interval training with the cardiovascular system and cardiac anti-aging.
\end{abstract}

Keywords: Cardiac anti-aging, cardiovascular system, cardiovascular disease, high intensity interval training.

\section{INTRODUCTION}

Cardiovascular diseases are classified as diseases of the blood circulation system and are noncommunicable diseases according to the classification of diseases such as the Household Health Survey and Basic Health Research conducted by the Health Department of the Republic of Indonesia. The results of the 2007 Riskesdas stated that the proportion of non-communicable diseases in Indonesia in 2007 was $60 \%$, twice as large as the proportion of infectious diseases $(28 \%)$. The main prevalence of noncommunicable diseases in 2007 was hypertension 29.8\%, diabetes mellitus $1.1 \%$ (based on diagnosis by health professionals and symptoms), and metabolic syndrome 18.8\%. Cardiovascular disease infection is the main source of death on this planet. Deaths caused by cardiovascular disease are more common in low and middle-income countries (82\%), including Indonesia. Based on data in Indonesia, cardiovascular disease causes more than $30 \%$ of deaths at all ages with the proportion of deaths due to: (i) stroke by $15.4 \%$, (ii) hypertension by $6.8 \%$, (iii) coronary heart disease by $5,1 \%$, and (iv) heart disease at $4.6 \% .^{1,2}$ Ischemic heart disease and stroke are the most common causes of cardiovascular disease in each country $\mathrm{B}^{3,4}$.

There are a few danger factors prompting the turn of events and movement of CVD, yet one of the most unmistakable is an inactive way of life. A sound way of life can be portrayed by both weight and reliably low degrees of actual movement. A few long-term researches have indicated that expanded actual action is related with a decrease taking all things together reason mortality and may build future, an eff ect which is firmly connected to a decrease in the danger of creating cardiovascular and respiratory illnesses. 
Accordingly, way of life intercessions that plan to increment actual action and decrease obesity are appealing helpful strategies to battle most non-congenital types of CVD. ${ }^{5,6}$

The normal life expectancy of the human populace is expanding around the world, generally on account of declining ripeness and expanding life span. It has been anticipated that, in 2035, almost one of every four people will be 65 years or more ${ }^{7}$. Epidemiological investigations plainly show that maturing itself is the significant danger factor for cardiovascular and cerebrovascular diseases. Most of the research endeavors on anticipation of these sicknesses have disregarded the components hidden heart and vascular impacts of aging, and have focused all things being equal, on the headway of mediations that target customary cardiovascular danger factors. ${ }^{8}$

The consequences for cardiorespiratory and muscle frameworks have driven researchers to think about its application in the field of cardiovascular diseases. HIIT is defined as high-intensity exercise for a defined period of time interspersed with rest periods (moderate light intensity or complete rest). The examples of this activity include cycling, running or walking on a treadmill. HIIT does not include resistance training. ${ }^{9}{ }^{10}$ Several studies have reported that HIIT is more superior compared to continuous moderate exercise (CME), in improving the function of the cardio-vascular system. In addition,there is an association between HIIT and increased telomerase activity which provides anti-aging effects ${ }^{11}$. Looking at the background above, this study was conducted to determine the correlation between HIIT with the cardiovascular system and anti-aging.

\section{METHODS}

a. Study design. This research method uses literature studies from various international journals. This method is used with a review of adding knowledge related to the topics discussed by summarizing the topic of discussion

b. Eligibility criteria. The literature used in this study meets the inclusion criteria that have been set, which is literature related to the effect of HIIT on the cardiovascular system and anti-aging, published in 2011 and above.

c. Procedure in choosing reference. Literature was obtained through the Google Scholar and Pubmed databases with keywords of high intensity interval training, cardiovascular system, and cardiovascular anti-aging. This method provides factual information or analysis related to review of relevant literature and then compares the results in an article.

\section{DISCUSSION}

a. High intensity interval training

A suitable reaction to an acute aerobic exercise stimulus requires robust and coordinated physiological growth from the aspiratory, respiratory, skeletal muscle, and cardio-vascular systems. In this situation, recuperation of the cardiovascular systems after exercise happens over a time of minutes to hours, during which numerous attributes of the framework, even how it is controlled, change after some time ${ }^{12}$. Any guidelines currently agree to recommend exercising at least 30 minutes a day at moderate intensity (walking, cycling, gardening) for the health of the cardiovascular system. However, this is difficult to do considering the patient's limited time. One of the techniques in exercise is cardio exercise or characterized as a concise, irregular bursts of vivacious movement, mixed by times of rest or low-force work out. HIIT alludes to an activity program that is portrayed by moderately short eruptions of fiery movement, mixed by times of rest or low-power practice for recuperation. HIIT is basically applied using in aerobic exercise, like cycling on an ergometer and running on a treadmill ${ }^{13}$. A proposed significant favorable position of HIIT is that it requires some investment than conventional moderate-intensity practice alternatives to get similar medical advantages. Even very small amounts of intense exercise improves health outcomes and reduce cardiovascular mortality risk ${ }^{14}$. The definition of HIIT varies across studies. Be that as it may, a few 
researches characterize HIIT as focused energy practice with the objective power existing in submaximal VO2max between $85 \%$ and $95 \%$ of the peak heart rate. HIIT may in this way show to an ideal technique for actualizing incredible exercise in people who are ill suited or not used to enthusiastic power actual movement. Figure 1 graphically portrays the example of activity in an average HIIT sessions ${ }^{15}$. Regularly, the term high-impact HIIT is utilized for HIIT with sub-VO2max intensity. The length of HIIT is characterized as 30s to a few minutes. Different lifestyle requires different HIIT exercise for people with related contaminations with or without cardiac diseases. Most use protocol is a $4 \times 4 \mathrm{~min}$, demonstrating positive rate of $70.2 \%$ in the coronary artery disease group and $75 \%$ in the CHF group. Different exercise with practice terms of $30 \mathrm{~s}, 2$ minutes, and 3 minutes were additionally compelling in a predetermined number of studies. The $4 \times 4 \mathrm{~min}$ protocol is prevalently utilized in patients with way of life related in addition to cardiovascular disease ${ }^{16}$. There are several modifications to HIIT. Matsuo et al ${ }^{17}$ in their research developed a new method for HIIT, namely The Japanese high-intensity interval aerobic training (J-HIAT) program, namely the 3 set method of 2-3 minutes cycling at vigorous intensity (1st and 2nd sets: 3 minutes at $85 \%-90 \%$ VO2peak, 3rd set: 3 min at $80 \%-85 \%$ VO2max) with 2-minutes active rest at 50\% VO2peak between each set. Another modification was made by Osuka et $\mathrm{al}^{18}$. Graphical representation of a typical HIIT protocol (figure 1). Horizontal axis is for time and vertical axis is for exercise intensity, expressed comparative with maximal high-impact limit (dotted line). The "on" portion of HIIT is normally $>70 \%$ of max $\mathrm{O} 2$ consuming limit, and these can last from only a couple seconds to a few minutes. ${ }^{15}$

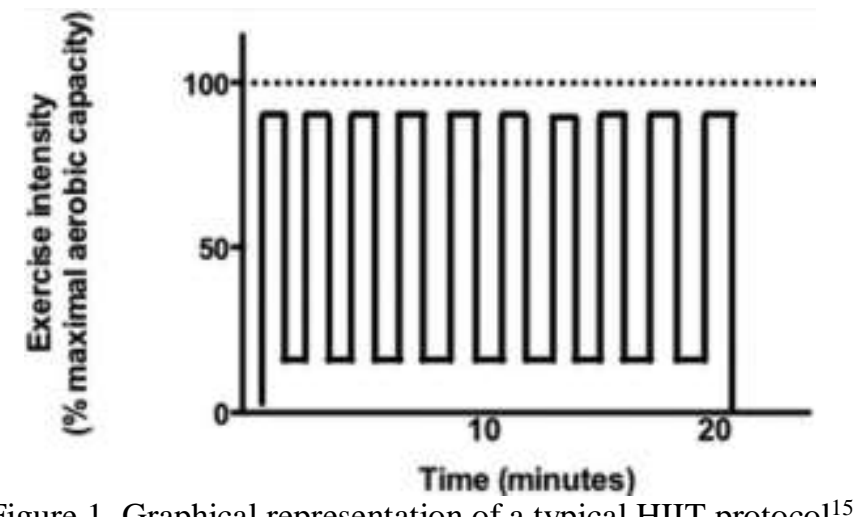

Figure 1. Graphical representation of a typical HIIT protocol ${ }^{15}$

The method of the elderly Japanese male version of high intensity interval aerobic training (EJ-HIAT) was established. This method includes 3 sets of cycling for 2-3 min when 75\% -80\% VO2peak (1st set: 3 min at $85 \%$ VO2peak, 2nd set: 2 min at $80 \%$ VO2peak, and 3rd set: 2 min at 75\% VO2peak) with 1-2-min active rest at 50\% VO2peak (first set: $2 \mathrm{~min}$, second set: $1 \mathrm{~min}$ ) (60-69-year-old sedentary elderly men; mean age, $67.6 \pm 1.8$ years). A bit by bit diminishing burden was anticipated $2-3 \mathrm{wk}$, focusing on the protocol portrayed previously. A critical metabolic and aerobic reaction was accomplished by the more limited convention than the $4 \times 4$ min convention with a completion rate of $100 \%$. However, in this study, the HIIT assessment was not carried out in detail.

\section{b. Cardiovascular system and High intensity interval training}

Of several systemic diseases, cardiovascular disease often uses high intensity interval training because it has beneficial and potential effects. Several effects of HIIT affect VO2max, heart rate, blood pressure and its safety. Meta-analysis conducted by $\mathrm{Cao}$ et al expects to straightforwardly look at the impacts of HIT and MICT preparing conventions for enhancements to cardiorespiratory system in youngsters and teenagers. This research is clear, HIIT is more likely to improve system function in children and adolescents 6-17 years in MICT. This study is supported by a study conducted by Maclnnis MJ et al where HIIT increased VO2max levels in obese children from 1.6 - $3.7 \mathrm{ml} . \mathrm{kg}-1 . \mathrm{min}-1$. From this research, it is stated that HIIT is a promising method because it has the advantage of being time-efficient in improving the outcome of the cardiorespiratory system. Some of the explanations may be given with respect to why 
the impact of HIIT on cardiorespiratory framework was superior to MICT. To begin with, mitochondrial transformations to transient preparing is a potential instrument. In the investigation by MacInnis et al, members performed six instructional courses with every leg more than about fourteen days, with one leg performing HIIT and the other leg performing MICT, and the volume of preparing was indistinguishable for every leg. The outcomes indicated that HIIT contrasted with MICT inspired a more noteworthy expansion in mitochondrial substance, and HIIT incited more prominent expansions in citrate synthase maximal movement, type II fiber activation, adenosine monophosphate enacted protein kinase action and mass-explicit JO2 (oxygen flux) comparative with MICT might be a contributing element. Second, HIIT protocol was more efficient on central adaptation, such as cardiac output, maximal stroke volume, and blood volume, which are important components of better outcome in the cardiorespiratory system ${ }^{19,20}$.

c. VO2peak and VO2max

Research conducted by Guiraud et $\mathrm{al}^{10}$ and Cornish et $\mathrm{al}^{21}$ analyzed the effects of HIIT with cardiac rehabilitation. From these two studies it is concluded that HIIT has the effect of increasing peak oxygen consumption (VO2peak). This study explains that individuals with the HIIT method can achieve a significant improvement in risk factors for cardiovascular disease compared to MCT (Moderate Continuous Exercise Training). Because VO2peak is such a strong predictor of cardiovascular disease morbidity and mortality, several studies have analyzed the relationship between HIIT affecting functional changes. Although the underlying mechanism is still not fully understood, some investigators have had explanations such as the rest periods that exist in HIIT allowing patients with cardiovascular disease to complete periods of exercise in a short period of time which is capable of providing a higher cardiac stimulus than $\mathrm{MCT}^{10}$, 16.

Another study conducted by Keech et al covered 29 patients with CAD completed 6-weeks $\times 2$ meetings for every seven days stretch) of HIIT within outpatient (phase 2 ). HIIT involved 15 repetitions $\times 30$-seconds cycling at $\sim 85-90 \%$ maximum heart rate, sprinkled with 30-seconds of dynamic recuperation. Key results covered patient wellbeing, adequacy (top high-impact limit, body creation, circulatory strain and vascular capacity), and patient adherence and satisfaction. From this study, it was found that the VO2 peak increased by an average of $12 \%$, equivalent to $3.1 \mathrm{MlO} 2 / \mathrm{kg} / \mathrm{min}$ or $1 \mathrm{MET} .{ }^{22}$

A Research conducted by Bacon et al explained the mechanism behind the increase in VO2max, explaining that this rest interval can increase cardiovascular capacity due to increased cardiac output as well as peripheral oxygen extraction. In addition, this study reveals the most effective HIIT technique in increasing VO2max is the "Hickson protocol", which incorporates 10 weeks of training, 6 days per week with stretch and nonstop training on substitute days. Span training comprises of six 5 minutes meetings on a cycle ergometer at a work rate drawing nearer the subjetc's VO2max. There are 2 minutes of active rest between periods. As the subject's capacity yield increments during preparing, the intensity is increased as needed. On the non-stretch days, persistent training comprises of running as quick as workable for $30 \mathrm{~min} / \mathrm{day}$ during the first week, $35 \mathrm{~min} /$ day during the subsequent week, and $40 \mathrm{~min} /$ day or longer from that point. This study notes that with this protocol, VO2max will continue to increase until week 10 of the program but this protocol is just utilized in people without a background marked by cardiovascular disease because it is very tiring. ${ }^{23,24}$

\section{d. Blood pressure}

The first study was conducted by Grace et al analyzed the effects of high intensity interval training on blood pressure, echocardiography, cardiac strain mechanics and maximal metabolic capacity in elderly. This study included 39 participants who went through 3 phases. The first phase is enrolment (Phase A), the second phase is pre-conditioning exercise (Phase B), and the last is HIIT once every 5 days before reassessment (Phase C). From this study, it was found that HIIT improves systolic blood pressure, mean arterial blood pressure, product rate pressure and heart rate reserve in the elderly. ${ }^{25,26}$ In the context of blood pressure, other studies conducted by Izadi et al also analyzed the effects of HIIT on blood pressure, apelin, and plasma 
NOx levels in elderly patients with hypertension. The study included 15 research subjects and 15 controls $(\mathrm{n}=30)$. The HIIT method used in this study is 3 sessions for 35 minutes per week $(1.5$ min interval at 85 $90 \%$ of heart rate reserve and 2 min active phase at 50-55\% of HRR). The study reviewed applein plasma, nitrate (NOx), and endothelin-1 (ET-1) before and after intervention. In this study also obtained the appropriate results namely HIIT increases levels of apelin and plasma NOx which leads to an improvement in blood pressure ${ }^{27}$. However, different results were obtained in the study conducted by Ramos et $\mathrm{al}^{28}$ and Astorino et $\mathrm{al}^{29}$. The meta-analysis conducted by Ramos et al included several studies that compared HIIT and MICT methods on cardiovascular system function. One of the variables assessed in this study is blood pressure. From this study, blood pressure was measured in a sitting position after a 5-15 minutes rest period. The majority of studies comparing blood pressure on HIIT and MICT techniques did not show a significant difference. However, there are 1 study that have shown a significant improvement in diastolic blood pressure after HIIT, although only slightly. Other studies in this meta-analysis revealed shorter duration of HIIT intervals but a higher frequency of showing improvement in diastolic blood pressure after the 12week program ${ }^{28}$. Ramos et al's research was supported by Astorino et al, who explained that resting and wandering BP shows up not to be impacted by practice intensity. This research included young ladies in danger of hypertension where there was no huge distinction in wandering pulse among HIIT and CMT. Nonetheless, this investigation likewise uncovered that HIIT was better than CMT in decreasing diastolic circulatory strain reaction to a reviewed practice following a time of about four months. This decrease may have significant ramifications for cardiovascular disease's visualization, as supported by the association between exaggerated BP response to exercise and rate of future hypertension and the more noteworthy BP reaction to exercise regularly found among this high danger populace ${ }^{21}$.

\section{d. Heart rate}

Resting heart rate or RHR was significantly associated with mortality. Research conducted by Gramer et al showed a huge decrease in pulse through yoga meditation where in this study it was concluded that yoga interventions reduce cardiovascular risk factors. This analysis explains that there is a decrease in pulse of $6.59 \mathrm{bpm}$ in examines that contrasted yoga and no-treatment regular consideration or any dynamic treatment in solid members. ${ }^{30,31}$ The study conducted by Schneider et al included 37 trained athletes (divided into the ST group with a total of 19 participants and the HIIT group with a total of 18 participants). This study assessed daily short- and long-term HR for a 4 days baseline period, a 6 day overload micro cycle, and a 4 day recovery period. From this study it was found that HIIT significantly reduced HR compared to ST in the short term, but when assessing the long-term effects there was no significant effect ${ }^{32}$. Another study conducted by Lino Sergio Rocha Conceição assessed the effect of HIIT on the cardiovascular system where one of the variables assessed was heart rate. From this study it is concluded that HIIT provides a significant improvement in peak heart rate and heart rate reserve compared to MICT. The study explains that HIIT improves peak heart rate compared to no exercise training. This study concludes that HIIT has a superior effect on peak heart rate and heart rate reserve than MICT. ${ }^{33}$

\section{f. Safety}

A study conducted by Wewege et al. Meta-analysis assessed adverse events arising from HIIT and MICT methods. From this study it was found that there were 7 adverse events reported in the entire study $(1,117$ participants). Five cases occurred during or after HIIT, and 2 cases occurred during or after MICT. Of the 7 adverse events, 2 were cardiovascular events and were associated with HIIT. One of them was classified as a non-fatal major event (ventricular arrhythmia leading to cardiac arrest, treated with direct current cardioversion, and the patient was effectively revived; occurred first week of training; member pulled out from the examination; the patient had rejected cardioverter - defibrillator implantation before consideration into the research); the other could be delegated minor (syncope during one instructional meeting; member proceeded in the examination). Of these two cardiovascular events occurred in the population with HF. The HIIT method that occurs during cardiovascular events involves $4 \times 4$ minutes intervals (with a 3 minutes rest period) and applying lower intensity (88\% HR peak). Of the 7, 5 were classified as non-cardiovascular events. Two of them complained of musculoskeletal related symptoms in 
the lower extremities where 1 incident occurred in the HIIT group and 1 incident occurred in the MICT group. The patient with HF encountered an unseemly implantable cardioverter-defibrillator release disconnected to arrhythmia happening during an activity meeting in the last seven day stretch of the HIIT and halted the activity program. One patient with HF experienced unsteadiness inside 3 hours after a managed HIIT meeting, with no perceptible cardiovascular reason, and the member proceeded with the preparation program with no reoccurrences. Based on the accessible preparing and AE information, a significant cardiovascular function happened at a pace of 1 for each 17083 HIIT meetings (11 333 preparing hours), while the general cardiovascular function rate for HIIT (counting minor functions) was 1 for every 8541 meetings (5667 preparing hours). MICT didn't incite any cardiovascular AE's. The general AE rate (every single revealed function) was 1 function for each 3417 meetings (2227 training hours) for HIIT and 1 function for each 7134 meetings (5606 training hours) for MICT. In general, the danger distinction for all AEs among HIIT and MICT are not significantly different. ${ }^{34}$

\section{g. Cardiac anti-aging}

Aging is related with a reformist reduction in physiological cycles this include an expanded danger of intricacies from specific sicknesses. Mature age is a significant danger factor for cardiovascular disease where there is chronic exposure to diabetes, hypertension, smoking, hypercholesterolemia, and other cardiovascular danger factors. Apart from old age, the internal mechanisms of aging also result in changes in the heart which lead to increased cardiovascular mortality and morbidity. ${ }^{35,36}$ There are several mechanisms underlying cardiac aging including mitochondrial dysfunction, deregulation of microRNA, cardiac stem- / progenitor cell aging, impaired calcium homeostasis, adverse extracellular matrix remodeling, alttered nutrient and growth signaling, and activation in neurohormonal signaling. However, in the literature this review is not explained further. ${ }^{37}$ Basic and utilitarian problem inside the hearts and arteries that obviously gotten show with regards to CVD have a transcendent part in the boundless issue inside the living being that goes with cutting edge age in light of the fact that the cardiovascular system is vital to the endurance of other organ system. In figure 2 we can see a model regarding cardiac aging. (portrayed beneath the clinical practice limit line) modify the substrate on which cardio-vascular sickness (elements over the line). In this way, elements over the line are as of now named "diseases" that lead to heart and brain failure. These changes by and by thought to happen because of a "normal aging process" are portrayed beneath the line, that adjust the substrates on which cardiovascular infection (elements over the line) is superimposed ${ }^{38}$.

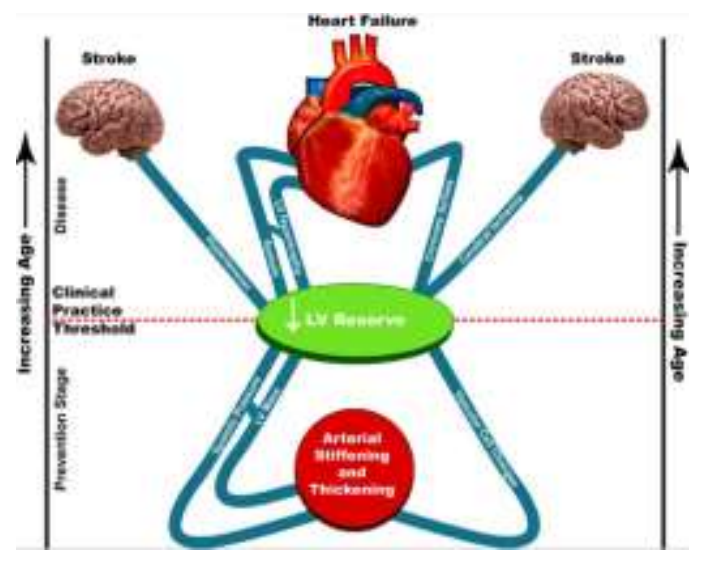

Figure 2. Changes because of aging in cardiac and vascular properties

One of the studies conducted by Soori R et al by analyzing the effect of HIIT on cardiac muscle apoptosis markers, which in this context is discussed are Bcl-2 and Bcl-xL, both of which regulate mitochondrial dysfunction and proapoptotic protein. Whereas in aging, both of these markers decreased significantly which led to cardiac aging. From this study, it was found that HIIT increases the expression 
of the Bcl-2 gene in mice which has a cardiac anti-apoptotic effect.. ${ }^{39}$ Another study by Vincent et al assessed whether there was a positive effect of 2-week HIIT on mitochondria. Eight guys played out a single-leg cycling protocol $(12 \times 1$ min intervals at $120 \%$ peak power output, 90 s recovery, 4 days / week). From this study, it was found that HIIT increases CS activity and absolute oxidative phosphorylation (OXPHOS) capacities. ${ }^{40}$ According to Chepelev NL, et al. aging, especially cardiac aging, is associated with decreased activity of citrate synthase. ${ }^{41}$

\section{CONCLUSIONS}

Ordinary exercise effectsly affects wellbeing in older adults. Exercise training can improve muscle work, increment high-impact limit, and lessen the frequency of cardiovascular functions and non-cardiovascular chronic disease, in this manner decreasing actual slightness and postponing actual dependance. From this literature review, it can be concluded that high-intensity interval training improves the function of the cardiovascular system. In addition, there is evidence that HIIT also provides anti-aging cardiac effects. However, future research is needed to be able to implement these findings. A good understanding of the HIIT technique is especially useful in terms of reducing mortality and morbidity.

\section{REFERENCES}

1. $\quad$ Penelitian B, Kesehatan P. Riset Kesehatan Dasar Laporan Nasional 2007.; 2007.

2. Prevention of Cardiovascular Disease Guidelines for Assessment and Management of Cardiovascular Risk WHO Library Cataloguing-in-Publication Data.; 2007. www.inis.ie

3. Ruan Y, Guo Y, Zheng Y, et al. Cardiovascular disease (CVD) and associated risk factors among older adults in six low-and middle-income countries: Results from SAGE Wave 1. BMC Public Health. 2018;18(1). doi:10.1186/s12889-018-5653-9

4. Sidney S, Quesenberry CP, Jaffe MG, et al. Recent trends in cardiovascular mortality in the United States and public health goals. JAMA Cardiol. 2016;1(5):594-599. doi:10.1001/jamacardio.2016.1326

5. Nystoriak MA, Bhatnagar A. Cardiovascular Effects and Benefits of Exercise. Front Cardiovasc Med. 2018;5. doi:10.3389/fcvm.2018.00135

6. Pinckard K, Baskin KK, Stanford KI. Effects of Exercise to Improve Cardiovascular Health. Front Cardiovasc Med. 2019;6. doi:10.3389/fcvm.2019.00069

7. Steenman M, Lande G. Cardiac aging and heart disease in humans. Biophys Rev. 2017;9(2):131-137. doi:10.1007/s12551-017-0255-9

8. Alfaras I, Di Germanio C, Bernier M, et al. Pharmacological Strategies to Retard Cardiovascular Aging. Circ Res. 2016;118(10):1626-1642. doi:10.1161/CIRCRESAHA.116.307475

9. Kessler HS, Sisson SB, Short KR. The potential for high-intensity interval training to reduce cardiometabolic disease risk. Sport Med. 2012;42(6):489-509. doi:10.2165/11630910-000000000-00000

10. Guiraud T, Nigam A, Gremeaux V, Meyer P, Juneau M, Bosquet L. High-intensity interval training in cardiac rehabilitation. Sport Med. 2012;42(7):587-605. doi:10.2165/11631910-000000000-00000

11. Gillen JB, Martin BJ, MacInnis MJ, Skelly LE, Tarnopolsky MA, Gibala MJ. Twelve Weeks of Sprint Interval Training Improves Indices of Cardiometabolic Health Similar to Traditional Endurance Training despite a Five-Fold Lower Exercise Volume and Time Commitment. Sandbakk $\varnothing$, ed. PLoS One. 2016;11(4):e0154075. doi:10.1371/journal.pone.0154075

12. Romero SA, Minson CT, Halliwill XR. The cardiovascular system after exercise. J Appl Physiol. 2017;122(4):925-932. doi:10.1152/japplphysiol.00802.2016

13. Feito Y, Heinrich K, Butcher S, Poston W. High-Intensity Functional Training (HIFT): Definition and Research Implications for Improved Fitness. Sports. 2018;6(3):76. doi:10.3390/sports6030076

14. Roy M, Williams SM, Brown RC, et al. High-Intensity Interval Training in the Real World: Outcomes from a 12-Month Intervention in Overweight Adults. Med Sci Sports Exerc. 2018;50(9):1818-1826. doi:10.1249/MSS.0000000000001642

15. Francois ME, Little JP. Effectiveness and safety of high-intensity interval training in patients with type 2 diabetes. Diabetes Spectr. 2015;28(1):39-44. doi:10.2337/diaspect.28.1.39

16. Ito S. High-intensity interval training for health benefits and care of cardiac diseases - The key to an efficient exercise protocol. World J Cardiol. 2019;11(7):171-188. doi:10.4330/wjc.v11.i7.171 
17. Matsuo T, Saotome K, Seino S, et al. Effects of a low-volume aerobic-type interval exercise on VंO $2 \mathrm{max}$ and cardiac mass. Med Sci Sports Exerc. 2014;46(1):42-50. doi:10.1249/MSS.0b013e3182a38da8

18. Osuka Y, Matsubara M, Hamasaki A, Hiramatsu Y, Ohshima H, Tanaka K. Development of low-volume, high-intensity, aerobic-type interval training for elderly Japanese men: a feasibility study. Eur Rev Aging Phys Act. 2017;14(1). doi:10.1186/s11556-017-0184-4

19. Cao M, Quan M, Zhuang J. Effect of high-intensity interval training versus moderate-intensity continuous training on cardiorespiratory fitness in children and adolescents: A meta-analysis. Int J Environ Res Public Health. 2019;16(9). doi:10.3390/ijerph16091533

20. MacInnis MJ, Zacharewicz E, Martin BJ, et al. Superior mitochondrial adaptations in human skeletal muscle after interval compared to continuous single-leg cycling matched for total work. J Physiol. 2017;595(9):29552968. doi:10.1113/JP272570

21. Cornish AK, Broadbent S, Cheema BS. Interval training for patients with coronary artery disease: A systematic review. Eur J Appl Physiol. 2011;111(4):579-589. doi:10.1007/s00421-010-1682-5

22. Keech A, Holgate K, Fildes J, Cummins L, Lewis C, Yu J. High-intensity interval training for patients with coronary artery disease: Finding the optimal balance. Published online 2020. doi:10.1016/j.ijcard.2019.09.060

23. Freyssin C, Verkindt C, Prieur F, Benaich P, Maunier S, Blanc P. Cardiac rehabilitation in chronic heart failure: Effect of an 8-week, high-intensity interval training versus continuous training. Arch Phys Med Rehabil. 2012;93(8):1359-1364. doi:10.1016/j.apmr.2012.03.007

24. Bacon AP, Carter RE, Ogle EA, Joyner MJ. VO2max Trainability and High Intensity Interval Training in Humans: A Meta-Analysis. Earnest CP, ed. PLoS One. 2013;8(9):e73182. doi:10.1371/journal.pone.0073182

25. Flint AC, Conell C, Ren X, et al. Effect of Systolic and Diastolic Blood Pressure on Cardiovascular Outcomes. N Engl J Med. 2019;381(3):243-251. doi:10.1056/nejmoa1803180

26. Grace F, Herbert P, Elliott AD, Richards J, Beaumont A, Sculthorpe NF. High intensity interval training (HIIT) improves resting blood pressure, metabolic (MET) capacity and heart rate reserve without compromising cardiac function in sedentary aging men. Exp Gerontol. 2018;109:75-81. doi:10.1016/j.exger.2017.05.010

27. Izadi MR, Ghardashi Afousi A, Asvadi Fard M, Babaee Bigi MA. High-intensity interval training lowers blood pressure and improves apelin and NOx plasma levels in older treated hypertensive individuals. J Physiol Biochem. 2018;74(1):47-55. doi:10.1007/s13105-017-0602-0

28. Ramos JS, Dalleck LC, Tjonna AE, Beetham KS, Coombes JS. The Impact of High-Intensity Interval Training Versus Moderate-Intensity Continuous Training on Vascular Function: a Systematic Review and MetaAnalysis. Sport Med. 2015;45(5):679-692. doi:10.1007/s40279-015-0321-z

29. Astorino TA, Allen RP, Roberson DW, Jurancich M. Effect of high-intensity interval training on cardiovascular function, VO 2max, and muscular force. J Strength Cond Res. 2012;26(1):138-145. doi:10.1519/JSC.0b013e318218dd77

30. Cramer H, Lauche R, Haller H, Steckhan N, Michalsen A, Dobos G. Effects of yoga on cardiovascular disease risk factors: A systematic review and meta-analysis. Int $J$ Cardiol. 2014;173:170-183. doi:10.1016/j.ijcard.2014.02.017

31. Reimers A, Knapp G, Reimers C-D. Effects of Exercise on the Resting Heart Rate: A Systematic Review and Meta-Analysis of Interventional Studies. J Clin Med. 2018;7(12):503. doi:10.3390/jcm7120503

32. Schneider C, Wiewelhove T, Raeder C, et al. Heart Rate Variability Monitoring During Strength and HighIntensity Interval Training Overload Microcycles. Front Physiol. 2019;10(MAY). doi:10.3389/fphys.2019.00582

33. Conceição LSR, Gois CO, Fernandes RES, et al. Effect of High-Intensity Interval Training on Aerobic Capacity and Heart Rate Control of Heart Transplant Recipients: a Systematic Review with Meta-Analysis. Brazilian J Cardiovasc Surg. 2020;(AHEAD):1-8. doi:10.21470/1678-9741-2019-0420

34. Wewege MA, Ahn D, Yu J, Liou K, Keech A. High- Intensity Interval Training for Patients With Cardiovascular Disease-Is It Safe? A Systematic Review. J Am Heart Assoc. 2018;7(21). doi:10.1161/JAHA.118.009305

35. Dai DF, Chen T, Johnson SC, Szeto H, Rabinovitch PS. Cardiac aging: From molecular mechanisms to significance in human health and disease. Antioxidants Redox Signal. 2012;16(12):1492-1536. doi: 10.1089/ars.2011.4179

36. North BJ, Sinclair DA. The intersection between aging and cardiovascular disease. Circ Res. 2012;110(8):1097-1108. doi:10.1161/CIRCRESAHA.111.246876

37. Chiao YA, Rabinovitch PS. The aging heart. Cold Spring Harb Perspect Med. 2015;5(9). doi:10.1101/cshperspect.a025148 
38. Lakatta EG. So! What's aging? Is cardiovascular aging a disease? J Mol Cell Cardiol. 2015;83:1-13. doi:10.1016/j.yjmcc.2015.04.005

39. Soori R, Ghram A, Zare Shahneh M, Choobineh S, Costa PB, Voltarelli FA. Effects of high intensity interval training and aging on cardiac muscle apoptosis markers in C57BL/6 Mice. Sport Sci Health. Published online September 21, 2020. doi:10.1007/s11332-020-00670-2

40. Vincent G, Lamon S, Gant N, et al. Changes in mitochondrial function and mitochondria associated protein expression in response to 2-weeks of high intensity interval training. Front Physiol. 2015;6(FEB):51. doi:10.3389/fphys.2015.00051

41. Chepelev NL, Bennitz JD, Wright JS, Smith JC, Willmore WG. Oxidative modification of citrate synthase by peroxyl radicals and protection with novel antioxidants. J Enzyme Inhib Med Chem. 2009;24(6):1319-1331. doi:10.3109/14756360902852586 